\title{
Development of the Child Needle Sensation Questionnaire (CNSQ)
}

\section{Abstract}

Objective: De qi sensations occur during acupuncture treatment and are believed to be important for a successful acupuncture therapy. Items addressing de qi sensations range from bruised, warm, and spreading to sharp, pricking and electric shock. How does age influence de qi sensations? In order to investigate this, we developed a de qi questionnaire for children.

Methods: We developed a pictographic sentence based de qi questionnaire for children the child needle sensation questionnaire (CNSQ) on the basis of the SNSQ. The first version contained 17 pictographic sentences and 25 items and was tested by means of a 1 to 1 connection test in 40 adults. In the second revised version of the CNSQ, items were reduced to 17 and it was retested in 49 adults. Subsequently we applied bilateral LI 4 Pyonex needle acupuncture to 20 adults and 20 school aged children and measured de qi sensations with the CNSQ. Pain was measured with a visual analogue numeric rating scale (NRS 11) 0-10.

Results: The most common de qi sensation in adults and children was spreading. Children chose fewer items 3, $2(\mathrm{SD}+/-)$ than adults $6,7(+/-) \mathrm{p}<0,05$. Children $0,95(\mathrm{SD}+/-0,95)$ and adults 1,2 (SD +/-1,15) reported similar pain on NRS 11 .

Conclusion: CNSQ could be used routinely in acupuncture trials in children to address specific and non-specific acupuncture effects related to acupuncture modalities and sites. Frequency und de qi sensations themselves seem to be strongly influenced by age and should be taken more into account before drawing conclusions from acupuncture trials with inhomogeneous age distribution.

Keywords: Acupuncture; Children; De qi; Questionnaire; Development

\section{Introduction}

Acupuncture is an accepted therapeutic method and well established within the integrative medicine. The treatment of pain remains one of the most studied indications for acupuncture therapy. Acupuncture pain research provided data that de qi sensations seem to be important for analgesia [1]. De qi is believed to be a sign for a successful connection of acupuncture needles to energy pathways of the body [2]. De qi sensations represent a predictor for a positive treatment outcome of acupuncture [3-6]. In order to understand de qi and its relevance for the efficacy and mechanisms of action of acupuncture standardized quantitative de qi scales have been developed. Vincent et al. and later Park et al. started derive these scales from pain questionnaires [7-9]. McPherson continued the development and created the SASS (subjective acupuncture sensation scale). Using the SASS McPherson found that numbness and soreness seem to be a positive predictive marker for the therapy effect of acupuncture $[10,11]$. After using the SASS for a couple of years the group published a revised version the MASS (Massachusetts General Hospital Acupuncture sensation scale). Finally White and colleagues introduced the SNSQ (Southampton Needle Sensation questionnaire) in 2008. They claimed that former instruments mainly derived from pain questionnaires and added qualitative research methods. They divided de qi in an aching and a tingling de qi. Additionally they measured single items that occurred during acupuncture, but where not closely related to de qi sensations [12]. Items addressing de qi sensations ranged from bruised, warm, spreading and tingling to sharp, pricking and electric shock. The age distribution of the participants reached from 18 to 90 years and the mean age was 51 years. The SNSQ was translated in German and trialled from Pach and colleagues. They proposed that the SNSQ is not able to discriminate between pain and de qi [13].

The de qi phenomenon is still not completely understood. The different aspects of de qi sensations might be due to different tissues and nerve fibers conveying the local and systemic effects of acupuncture therapy [2]. Especially in modern acupuncture research like f-MRI or placebo controlled randomised studies de qi sensations should be recorded. As we could not find standardized quantitative de qi questionnaires for children we developed the child needle sensation questionnaire (CNSQ) on the basis of the SNSQ to investigate age specific influences of de qi sensations.

\section{Methods}

\section{Development of questionnaire}

We used the German version of the SNSQ by Pach and colleagues. We created sentences that pictographic describe the 
properties of a specific item for every of the 17 SNSQ items. For some difficult and problematic items like "Blauer Fleck" English version "bruised" we added one extra synonym to enhance better understanding. We ended up with 17 sentences and 25 Items. Forty adults were asked to link the pictographic sentences to the best fitting item. Per sentence one item could be chosen only once. In a second step we revised ambiguous pictographic sentences and reduced the numbers of items back to 17 according to the results of the first version and the original metric outline of the SNSQ. Again 49 adults connected the pictographic sentences to the best fitting items in the revised version of the CNSQ.

Does It feels like....

You bumped yourself and grazed something.

Pain you cannot point at with your finger.

Your arms or legs become heavy

you get hurt by boiling water

water is dropped

Water you are very uncomfortable

Pain you can only feel deep inside

A halfway blown up ballon at your mouth

A radiator in winter time

Something radiating from a point

You sat a long time with bended legs and afterwards they hurt

As if ants are crawling all over your hand

Something has stung you.

As if you had stubbed yourself and the pain is easing slowly

As if you are a door and somebody would constantly knock

Touching a hedgehog or walking barefoot over spiky stones

The cutting side of a knife

Sparkling, this happens sometimes when you touch somebody

\section{Measuring of de qi sensations and acupuncture}

We examined de qi sensations in adults and children on the basis of the CNSQ (Figure 1). We elicited de qi by performing acupuncture bilateral at LI 4 (Hegu) with indwelling Pyonex needles $0,2 \mathrm{x} 0,6 \mathrm{~mm}$ from Seirin. LI 4 is located on the dorsum of the hand, between the 1st and 2nd metacarpal bones, in the middle of the 2nd metacarpal bone on the radial side. Pyonex needles were stimulated simultaneously for 1 minute and afterwards we interviewed adults and children with the CNSQ. Acupuncture was performed through the same licensed medical doctor in all adults and children.

\begin{tabular}{|c|c|c|c|}
\hline None & Slight & Moderate & Intense \\
\hline & & & \\
\hline & & & \\
\hline & & & \\
\hline & & & \\
\hline & & & \\
\hline & & & \\
\hline & & & \\
\hline & & & \\
\hline & & & \\
\hline & & & \\
\hline & & & \\
\hline & & & \\
\hline & & & \\
\hline & & & \\
\hline & & & \\
\hline & & & \\
\hline & & & \\
\hline & & & \\
\hline & & & \\
\hline & & & \\
\hline & & & \\
\hline & & & \\
\hline & & & \\
\hline & & & \\
\hline & & & \\
\hline & & & \\
\hline
\end{tabular}

Figure 1: CNSQ - child needle sensation questionnaire. 


\section{Pain assessment}

We used a visual analogue scale for children with a numeric rating scale $11(0-10)$ to measure pain.

\section{Statistics}

All analyses were done with IBM SPSS Statistics 18 (SPSS Inc., an IBM Company, and Chicago IL, USA).

\section{Approval ethic committee}

The Ethic Committee of the Carl Gustav Carus University Hospital - Technical University Dresden approved this study.

\section{Results}

\section{Development of questionnaire}

The first version was tested in 40 adults. The distinctive feature was the connection of 17 pictographic sentences to the best fitting item out of 25 presented items. In the first version of the CNSQ sentences for the items warm, pricking, throbbing and stinging were recognized by more than $70 \%$ of the tested adults.
Between $50-70 \%$ of our test persons identified items fading, tingling, twinge, spreading, pressure, deep ache and heavy. The most difficult items to detect from our sentences were sharp, numb, hidden pain and bruised. The worst identification rate with $37,5 \%$ of the participants showed hidden pain.

To discriminate between two items with similar meanings e.g. dull ache and hidden pain we analysed item recognition results for the first 2 most frequent connections of the participants between pictographic sentence and item. Items were then identified over $80 \%$ for sensations electric shock, pricking, throbbing, twinge, tingling, warm, pressure, deep ache, uncomfortable and stinging. A recognition rate of $60-80 \%$ showed items sharp, fading, numb, spreading, heavy, hidden pain and bruised. The worst identification rate showed the item bruised with $62,5 \%$.

According to these results we revised our questionnaire in two ways. We reduced the items from 25 to 17 . Secondly we adapted some sentences to raise the discriminatory power. The revised version of the CNSQ questionnaire was tested in 49 adults. Finally the mean item identification rate achieved by participants was above $90 \%$ (Figure 2).

\begin{tabular}{|c|c|c|}
\hline $\begin{array}{l}\text { Does it feel likc ... } \\
\text { Aching det i }\end{array}$ & Item & \\
\hline 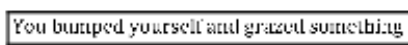 & orujsed & 67,0 \\
\hline Pailn you cannot point at with your fir.ger & hidden pain & 85,8 \\
\hline L.ifting something with 3 lot of weizht & hespy & 100 \\
\hline 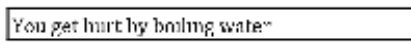 & stumping & 43.9 \\
\hline You are ver'y uncomfontahle & uncomfortible & 91,9 \\
\hline Pain you ram mily feeel inside & defp ache & $\mathrm{B} \cdot \mathrm{s}, \mathrm{B}$ \\
\hline 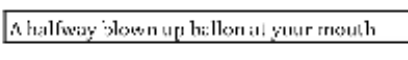 & gregsurs: & 75,11 \\
\hline \multicolumn{3}{|l|}{ Tingling dequ1 } \\
\hline A radi.tur in winter lime: & PMWn & 413 \\
\hline As if something radiates frono onc point & spyreading & 77,6 \\
\hline Fou sst a long time with bendied legs. & gुone csiép & 91,9 \\
\hline As if ants sre rumning all over your hand & tirglivis & 93.9 \\
\hline A mosquitts has stung you. & nwinge & 81.7 \\
\hline $\begin{array}{l}\text { As if you had sthlhbed yourself and the } \\
\text { prain is ens:ng slowily }\end{array}$ & fadixp & 91,9 \\
\hline 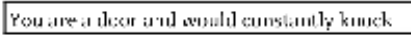 & thouligisiti & 91,9 \\
\hline \multicolumn{3}{|l|}{ Separale } \\
\hline Wialkiug bartefun uree spiky sloues & prickings & 31,7 \\
\hline The cutting sitte of a kaite & sharp & 100 \\
\hline $\begin{array}{l}\begin{array}{l}\text { Sparsling which som.ctimes hajpers swhen } \\
\text { you touch somebotty }\end{array} \\
\end{array}$ & electric shock & 100 \\
\hline LDR = iteres cetectiont rate jat "si. & Meaz & 90,2 \\
\hline
\end{tabular}

Figure 2: Children de qi questionnaire revised version. 


\section{De qi measurement with the CNSQ - Adults}

20 adults received acupuncture with Pyonex needles bilaterally at LI 4 and after stimulation completed the revised sentence questionnaire rating each de qi sensation sentence synonym CNSQ with a 4 item Likert scale $(0=$ none, $1=$ slight, $2=$ moderate and $3=$ intense). The mean age was 23,5 years $(S D+/-2,7)$ and gender distribution 8 male to 12 female. Participants tended to score towards the low end. The most frequent items revealed to be spreading, stinging, twinge and deep ache (Table 1). The mean VAS pain score on NRS 0-11 was 1, 2 (SD +/- 1, 15).

Table 1: The most frequent items revealed to be spreading, stinging, twinge and deep ache.

\begin{tabular}{|c|c|c|c|}
\hline Item & Adults, $\mathrm{n}=\mathbf{2 0}$ & Children, $\mathrm{n}=\mathbf{2 0}$ & Difference \\
\hline Aching sensations & MSS & MSS & 0,26 \\
\hline Bruised & 0,25 & 0,05 & 0,2 \\
\hline Hidden pain* & 0,65 & 0,25 & 0,4 \\
\hline Heavy & 0,2 & 0,2 & 0 \\
\hline Stinging* & 0,9 & 0,3 & 0,6 \\
\hline Uncomfortable* & 0,35 & 0,05 & 0,3 \\
\hline Deep ache* & 0,8 & 0,3 & 0,5 \\
\hline Pressure & 0,1 & 0,1 & 0 \\
\hline Tingling sensations & MSS & MSS & 0,3 \\
\hline Warm & 0,25 & 0,4 & $-0,15$ \\
\hline Spreading & 1 & 0,8 & 0,2 \\
\hline Gone asleep & 0,35 & 0,05 & 0,3 \\
\hline Tingling & 0,6 & 0,45 & 0,15 \\
\hline Twinge & 0,85 & 0,3 & 0,55 \\
\hline Fading & 0,55 & 0,3 & 0,25 \\
\hline Throbbing* & 0,55 & 0,1 & 0,45 \\
\hline Single items & MSS & MSS & 0,42 \\
\hline pricking** & 0,6 & 0,05 & 0,55 \\
\hline Sharp & 0,6 & 0,15 & 0,45 \\
\hline Electric shock & 0,6 & 0,35 & 0,25 \\
\hline
\end{tabular}

${ }^{*} \mathrm{p}<0,05 ;{ }^{* *} \mathrm{p}<0,001$

MSS: Mean Sum Score

\section{De qi measurement with the CNSQ - Children}

We trailed 20 school children mean age 11,1 years $(+/-3,2)$ with our CNSQ after they had received bilateral acupuncture at LI 4 with Pyonex needles. Children used to rate to the lower end of the Likert scale. Children never reported bruised and pricking. Children gave account to significantly fewer items than adults.
Children chose items warm and heavy more often than adults to describe de qi sensations. Adults and children reported similar results for spreading, pressure and fading (Table 1). Children indicated Pyonex acupuncture to be nearly pain free with a mean score of $0,95(+/-0,95)$ on visual analogue NRS 11 . Adults reported significantly more items namely 6,7 items and children 3,2 items (Figure 3).

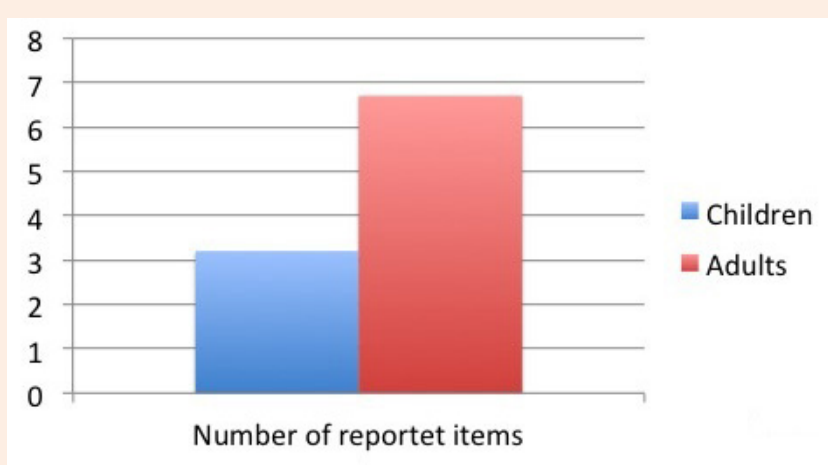

Figure 3: Number of reported items.

\section{Discussion}

Our study supports other trials that the SNSQ is able to detect de qi sensations. It contains 17 very closely related items, often possessing similar meaning. This fact made it difficult to find appropriate pictographic sentences describing this detailed sensations adequately. Pach and colleagues found more than $50 \%$ of the items were rated to be absent [13]. Our results in adults did not point at specific items that were not felt during Pyonex needle acupuncture at LI 4. De qi sensations seem to be very individual and interfere with person's experiences. No child detected the items bruised and pricking. Children reported de qi sensations warm and heavy more often than adults. These results might be due to the fact that children a more familiar with these sensations. Analogically to children projecting different pain locations primarily into the stomach. The most frequent de qi sensation spreading was comparable in children and adults. This is a very interesting finding. Imagination and expectation are able to highly influence perception, arousing the question whether spreading is truly felt, thought to be felt or both. De qi interaction effects seem to concern acupuncture site, mode of acupuncture, laterality, needle manipulation and prior acupuncture experiences. Our test persons received acupuncture bilaterally with Pyonex needles at LI 4. Needles were stimulated by simultaneously pressing the acupuncture stickers for one minute. All adults and children with the exception of one adult had not received acupuncture before.

Therefore our results are limited to bilateral Pyonex needle acupuncture at LI 4 and healthy acupuncture inexperienced adults and children.

Some researchers promote the argument that de qi research can only be performed in acupuncture experienced participants. We strongly disagree with this. Acupuncture experienced participants may be conditioned and we presume that they are less flexible and sensible due to their experience. In order to gain a greater picture and variety of de qi sensations we aimed on 
acupuncture naive participants. Taking the risk that they might lack experience in depicting de qi sensations. Research showed that de qi sensations seem to be conveyed through different nerve fibres. More over exist specific local effects or mediators for instance Substance P or CGRP, effects on spinal chord segments and systemic effects. There are studies showing important involvement of endorphins and the autonomous nerve system in mechanisms of action and the effect of acupuncture.

According to the results de qi research should focus on acupuncture specific and unspecific effects. The relation of different de qi sensations to certain nerve fibres and mediators should be studied. Additionally de qi questionnaires could be considered as standard content in every clinical acupuncture study.

An unspecific effect of acupuncture therapy seems to be age. Combining the results of the SNSQ and our CNSQ study we get strong evidence, thatage directly influences the recognition and the character of de qi sensations. Over $50 \%$ of the participants, mean age 51 years (range 18-90), in the SNSQ trial chose pricking, dull ache and sharp to describe their de qi sensation [12]. Nearly $2 / 3$ of these participants rated these items as painful. Unfortunately study data does not give information on age dependency. In contrast our age homogeneous adult group, mean age 23, 5 years $(+/-2,7)$ most frequently reported spreading and hardly no pain. Presumably humans tend to become more pain sensible with increasing age [14]. If you combine these results with findings from placebo research, that painful interventions have a greater therapeutic effect. SNSQ and our data suggest that acupuncture trials should be age controlled. Acupuncture studies often reveal a wide age range, due to recruitment difficulties and still missing general acceptance. The routine use of de qi questionnaires could contribute to a better detection and understanding of specific, unspecific and psychological effects of acupuncture regarding its efficacy.

The visual analogue NRS 11 results for Pyonex acupuncture at LI 4 from this study confirm studies of Anders et al., that Pyonex acupuncture is a nearly pain free and a suitable acupuncture modality in children and adults $[15,16]$. Interestingly we found no differences in pain intensity between children and adults.

The CNSQ needs to be validated separately for other acupuncture modalities, sites and disease specific acupuncture therapies. Limits of our study are the small sample size and that the de qi questionnaire was tested solely after acupuncture without pre testing. Furthermore the results are limited to bilateral LI 4 and Pyonex needle acupuncture.

\section{Conclusion}

The CNSQ is the first quantitative de qi questionnaire for children. De qi sensations seem to be an essential part to understand effects of acupuncture and should be recorded in every acupuncture trial. There are strong hints that age influences the frequency and quality of de qi sensations. Examining acupuncture therapies in homogeneous and age-controlled study designs might be beneficial. Younger adults and children seem to perceive similar pain intensities. Children tend to report significant fewer de qi sensations than adults.

\section{Contributor ship statement}

Conception \& Conducting EA; Development of questionnaire EA, CV; Drafting EA, MR; Revision MR, CV.

\section{References}

1. Chiang CY, Chang CT, Chu HL, Chu Hsiu-Ling, Yang Lian-Fang (1973) Peripheral afferent pathway for acupuncture analgesia. Sci Sin 16: 210-217.

2. Kong J, Gollub R, Huang T, Polich G, Napadow V, et al. (2007) Acupuncture de qi, from qualitative history to quantitative measurement. J Altern Complement Med 13(10): 1059-1070.

3. Takeda W, Wessel J (1994) Acupuncture for the treatment of pain of osteoarthritic knees. Arthritis Care Res 7(3): 118-122.

4. Berman BM1, Lao L, Langenberg P, Lee WL, Gilpin AM, et al. (2004) Effectiveness of acupuncture as adjunctive therapy in osteoarthritis of the knee: A randomized, controlled trial. Ann Intern Med 141(12): 901-910.

5. Scharf HP, Mansmann U, Streitberger K, Witte S, Krämer J, et al. (2006) Acupuncture and knee osteoarthritis: A three-armed randomized trial. Ann Intern Med 145(1): 12-20.

6. WittC, Brinkhaus B, Jena S, Linde K, Streng A, et al. (2005) Acupuncture in patients with osteoarthritis of the knee: A randomised trial. Lancet 366(9480): 136-143.

7. Vincent CA, Richardson PH, Black JJ and Pither CE (1989) The significance of needle placement site in acupuncture. J Psychosom Res 33(4): 489-496.

8. Park H, Park J, Lee H, Lee H (2002) Does De qi (needle sensation) exist? Am J Chin Med 30(1): 45-50.

9. Park J, Park H, Lee H, Lim S, Ahn K, et al. (2005) De qi sensation between the acupuncture experienced and the naive: A korean study II. Am J Chin Med 33(2): 329-337.

10. MacPherson H, Asghar A (2006) Acupuncture needle sensations associated with de qi: a classification based on experts' ratings. J Altern Complement Med 12(7): 633-637.

11. Kong J, Fufa DT, Gerber AJ, Rosman IS, Vangel MG, et al. (2005) Psychophysical outcomes from a randomized pilot study of manual, electro, and sham acupuncture treatment on experimentally induced thermal pain. J Pain 6(1): 55-64.

12. White P, Bishop F, Hardy H, Abdollahian S, White A, et al. (2008) outhampton needle sensation questionnaire: development and validation of a measure to gauge acupuncture needlesensation. J Altern Complement Med 14(4): 373-379.

13. Pach D, Hohmann C, Lüdtke R, Zimmermann-Viehoff $F$, et al. (2011) German translation of the Southampton Needle Sensation Questionnaire: use in an experimental acupuncture study. Forsch Komplementmed 18(6): 321-326.

14. Duracinsky M, Paccalin M, Gavazzi G, El Kebir S, Gaillat J, et al. (2014) ARIZONA study: is the risk of post-herpetic neuralgia and its burden increased in the most elderly patients?. BMC Infect Dis 14: 529.

15. Anders EF, Findeisen A, Lode HN, Usichenko TI (2012) Acupuncture for treatment of acute vomiting in children with gastroenteritis and pneumonia. Klin Padiatr 224(2): 72-75.

16. Anders EF, Findeisen A, Nowak A, Rüdiger M, Usichenko TI (2012) Acupuncture for treatment of hospital-induced constipation in children: a retrospective case series study. Acupunct Med 30(4): 258260 . 